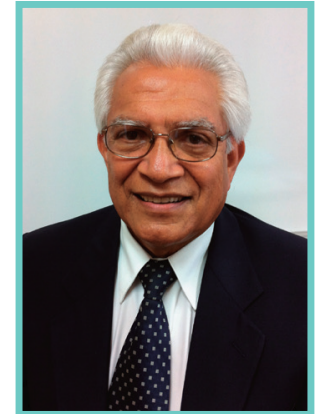

Agustín Campos Arenas

\title{
INNOVACIONES EN LA EDUCACIÓN PERUANA
}

\section{RESUMEN}

El presente artículo se centra en el tema de las innovaciones que se han realizado en la educación peruana. El autor presenta cuatro intentos de innovar la educación del país. Uno de ellos que afectó a todo el Sistema Educativo (Reforma Educativa). Otro, que se ocupó de la acción pedagógica y didáctica en las escuelas (DCN)

y, finalmente, dos que planteaban propuestas peruanas de enfoques internacionales (Bachillerato Peruano, Educación a Distancia).

\section{PALABRAS CLAVE}

Innovación educativa, sistema educativo peruano, propuestas de innovación.

\section{ABSTRACT}

This article focuses on the innovations that have taken place in peruvian education. The author presents four attempts to innovate education in the country. One that affected the entire education system (educational reform). Another, focuses in the pedagogical and didactic action in schools (DCN) and finally two proposals: high school and distance education.

\section{KEYWORDS}

Educational innovation, peruvian education system, innovation proposals.

\section{INTRODUCCIÓN}

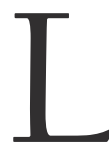

a Educación, como cualquier otra actividad humana, está sujeta al cambio y la actualización para responder L positivamente a las exigencias de los nuevos tiempos y de la sociedad en general. Estos cambios son cada vez más frecuentes, por la misma dinámica del conocimiento y del desarrollo humano.

Un cambio importante está representado por la Innovación, pues esta permite no solo avanzar; sino, también, acortar distancias hacia una situación declarada como ideal.

Enesteartículoseanalizan algunasinnovacioneseducacionales que se han planteado en el sistema educativo peruano a partir de 1970, tratando de explicar sus alcances y logros.

\section{INNOVACIÓN}

Es un término de uso frecuente en la actualidad por los actores de las diferentes áreas del saber o campos profesionales. Por ejemplo, Michael Porter (en Web and Macros, 2015) afirma que: La competitividad de una nación depende de la capacidad de su industria para innovar. El diccionario de la Real Académica de la Lengua Española (2014) menciona que proviene del latín innovatio -onis, que significa, en su acepción general, "creación o modificación de un producto". 
Otras definiciones interesantes al respecto son:

- Aquel cambio que introduce alguna novedad o varias en un ámbito, un contexto o producto (definición Abc).

- Motor que transforma problemas e ideas en valor (Web and Macros, 2015).

- Es un conjunto de ideas, procesos y estrategias más o menos sistematizados, mediante los cuales se trata de introducir y provocar cambios en las prácticas educativas vigentes... su propósito es alterar la realidad vigente, ... va asociada al cambio (Rimari Arias).

De acuerdo con las definiciones anteriores, una innovación puede conducir a alterar / modificar una situación / proceso / producto, existentes o presentar una totalmente nueva. Los autores la relacionan con el cambio. No obstante, hay que tener presente que toda innovación representa un cambio; pero, todo cambio no necesariamente representa una innovación.

La Innovación es un cambio previsto, planificado y ejecutado para mejorar una situación particular. Es un esfuerzo intencional o deliberado (Rimari Arias). La innovación puede darse en el producto, en el proceso, en la organización, en la exposición y mercadeo, entre otros.

Los autores han planteado una serie de tipologías relacionadas con las innovaciones. Así por ejemplo:

- Web and Macros, menciona la siguiente clasificación:

- Según su grado de originalidad

1. Radical, que conlleva a un nuevo producto o servicio, dejando de lado lo anterior.

2. Incremental, que implica mejoras graduales en un producto o servicio, a la manera del Kaisen Japonés.
- Según su aplicación

1. De producto, cuando el objeto de la innovación es un producto.

2. De servicio, cuando el objeto de la innovación es un servicio.

- Rimari Arias, presenta la clasificaciones siguientes:

- Según Modo

1. Adición, cuando la innovación incrementa "algo" a lo ya existente.

2. Reforzamiento, cuando se añade o "apuntala" algo en lo existente.

3. Eliminación, cuando la innovación concluye en que es necesario "separar", "eliminar" algo del producto o servicio.

4. Sustitución, cuando un aspecto/ elemento es reemplazado por algo nuevo.

5. Alteración, cuando se modifica en aspecto / elemento / proceso del ya existente.

6. Reestructuración, cuando se "rediseña" un proceso o producto obteniéndose una nueva propuesta.

- Según intensidad de cambio

1. Marginales, que conllevan ligeros cambios a la situación actual.

2. Adicionales, que se añaden a lo existente.

3. Fundamentales, que implican un cambio sustantivo o un nuevo producto / proceso / servicio.

- Según amplitud

1. Individuales, que afectan solo a un ente particular (un aula, un docente, etc.).

2. Institucionales, que tienen implicancias en toda la organización / sistema. 
- Strutton, Lumpkin y Vittel (1982) presentan la clasificación siguiente:

1. Continua o de mejoramiento permanente, en las que el cambio es pequeño.

2. Continua dinámica, en donde el cambio es mayor; pero, sin modificar sustantivamente la estructura del hecho / producto / servicio.

3. Discontinua, la que conlleva a un nuevo producto o servicio.

Rogers y Shoemaker (1971) afirman que la adopción de las innovaciones está sujeta a una serie de factores, tales como:

1. Ventaja competitiva; representada por la percepción de las personas acerca si el nuevo producto / servicio "supera" al existente.

2. Compatibilidad, en el sentido de que lo "nuevo" se "ajusta" a lo establecido.

3. Complejidad, relacionando en el grado de dificultad en la comprensión y uso de la innovación.

4. Prueba previa, que implica que puede ser "usado" en condiciones de prueba para familiarizarse y emprenderlo.

5. Visibilidad, que implica que los resultados de innovación sean visibles/observables.

\section{ALGUNOS CASOS}

Existe una serie de experiencias innovadoras de diferente magnitud que se han dado en el sistema educativo peruano. Algunos de estos casos se presentan a continuación.

\section{REFORMA EDUCATIVA}

Durante el gobierno militar (1968 - 1980) se llevó a cabo la "Reforma de la Educación", entre otras reformas, con el fin de dirigir al país a la creación de una nueva sociedad y, por ende, de un nuevo hombre. Fue un proceso muy amplio, que movilizó a muchos sectores del país y miembros de la comunidad educativa. Todo eso se plasmó en la nueva Ley General de Educación № 19326, en el año 1972.

Fue considerada "original", creativa e innovadora al proponer una reforma global del sistema y poner al individuo como el fin y no un medio (Campos, 2006). Mereció el reconocimiento nacional e internacional. Introdujo cambios profundos en lo académico y en lo administrativo.

Pero, fue el mismo gobierno militar, en su segunda fase, gobierno del general Francisco Morales Bermúdez, quien comienza "desactivar" la reforma educativa.

En el año 1980, el gobierno democrático del Arquitecto Fernando Belaúnde Terry propone una nueva Ley General de Educación (№ 23384), promulgada en 1982, que deja de lado muchas de las propuestas novedosas de la Reforma Educativa de los años 70.

\section{BACHILLERATO PERUANO}

El Bachillerato Internacional (BI), es un sistema especial aplicado en los últimos años/grados de la educación secundaria. Lo aplican algunos colegios privados del país y se caracteriza por su exigencia y el alto nivel y actualidad de los contenidos propuestos.

Está administrado por un organismo cuya sede está en Europa. Las Instituciones Educativas, de los diferentes países, que desean implementarlo en sus colegios deben registrarse, pagar los montos correspondientes y someterse a la normatividad del sistema de Bachillerato Internacional.

En los últimos años de la década de los años 90, el Ministerio de Educación se propuso generar un sistema similar al BI, para los centros estatales del país. El esfuerzo devino en el "Bachillerato Peruano" para los dos últimos 
grados de la secundaria. Se determinaron los contenidos curriculares, se prepararon los libros para cada área curricular, se capacitó a los docentes y se implementó en diferentes colegios del país.

A partir del año 2000, el nuevo gobierno deja de lado lo avanzado y se regresa a la secundaria convencional.

PROGRAMA DE EDUCACIÓN SECUNDARIA A DISTANCIA PARA ÁREAS RURALES

Uno de los problemas serios que tienen los Ministerios de Educación de los diferentes países, es proveer educación en áreas rurales donde la población no está concentrada sino dispersa.

Asimismo, se encuentran en espacios de difícil acceso y con pocos estudiantes para cada grado. A ello se le suma el hecho que los docentes profesionales no están dispuestos a ir a trabajar en dichas zonas.

Una solución ingeniosa, efectiva y práctica fue dada en México: crearon la "telesecundaria". Un sistema de instrucción que hace uso de: (1) televisión, (2) libro, (3) cuaderno de trabajo, (4) guía del participante. A falta de docentes profesionales, se cuenta con monitores (tutores) en el aula quien conduce la sesión de clase según lo prescrito en la guía de clases del tutor.

Los tutores son capacitados y administran todos los cursos de un grado. Para cada clase existe un pequeño programa de televisión, una lectura, y tareas en el cuaderno de trabajo. Se hace uso del satélite y de una antena parabólica. México "exportó" su modelo a otros países.

Esta idea se trajo al Perú a fines de los años 90. La licenciada Irene Bär, responsable del INTE (Instituto de Teleducación) juntó a un equipo de profesionales (Agustín Campos, Elisa Perea, Nelly Aliaga, Carlos Castillo) para que analice la posibilidad de adquirir los derechos para aplicar la Telesecundaria en el Perú.

El equipo, sin embargo, propuso hacer uso de la idea y crear un sistema propio que responda a los principios modernos del aprendizaje y con materiales contextualizados a la realidad peruana. Bajo la conducción del equipo antes mencionado se creó así un sistema de Educación a Distancia haciendo uso de los mismos elementos de la telesecundaria mexicana.

Se contrató a especialistas en cada una de las áreas curriculares y se prepararon prototipos de los materiales. Luego se inició el proceso de ubicación de los telecentros y se comenzó con $1^{\circ}$ de secundaria, en diferentes partes del país, donde esta opción representaba quizás su única posibilidad. Se aplicó durante los años siguientes $\left(2^{\circ}\right.$ y $3^{\circ}$ de secundaria), pero el gobierno de turno fue reemplazado por otro y ocurrió lo mismo que en el caso del Bachillerato.

\section{DISEÑO CURRICULAR NACIONAL (DCN)}

Del 2001 al 2011 el Ministerio de Educación emprendió la tarea de tener un solo documento curricular actualizado, consensuado con los docentes, validado, corregido tantas veces como fuera posible. Así, se llegó a tener varias versiones de dicha propuesta. En el 2011 se contaba con el documento curricular, ampliamente trabajado.

El nuevo gobierno (2011-2016) considera que hay otras maneras de entender los propósitos educacionales y la manera de hacerlos llegar y usar en las escuelas. Así se plantea dejar de lado el DCN y en su lugar hacer uso de nuevas propuestas: (1) Aprendizajes Básicos, (2) Rutas de Aprendizaje y (3) Mapas de Progreso.

Este cambio, repentino en algo diferente al DCN, causó dificultades en los docentes, lo cual ha llevado que el nuevo Ministro de Educación haya considerado evaluar y 
reorientar estos cambios. Lo cierto es que el DCN, ya no representa el documento orientador de la creación pedagógica en la educación peruana.

\section{CONCLUSIONES}

Se han presentado cuatro intentos de innovar la educación del país. Una de ellas que afectaba a todo el Sistema Educativo (Reforma Educativa). Otra que normaba la acción pedagógica y didáctica en las escuelas (DCN). Dos que planteaban propuestas peruanas de enfoques internacionales (Bachillerato Peruano,
Educación a Distancia) que apuntaban por un lado a mejorar la educación secundaria y a extender la educación secundaria a sectores no atendidos.

La innovación, para ser tal, debe ser duradera con aceptación, uso amplio y relacionado con una mejor práctica educativa. Sin embargo, en los casos presentados, a pesar que estos criterios se cumplían, consideraciones de tipo político-partidario llevaron a dejar de lado avances y cambios para empezar nuevamente con otras propuestas. La continuidad en el sector educación no es una de sus fortalezas.

\section{REFERENCIAS}

Campos Arenas, A. (2006). Reflexiones con dos maestros: Robert M. Gagné y Sydney R. Grant. Educación, 12, 51-54.

Definición abc. Tu diccionario hecho fácil. Recuperado de http:// www.definicionabc.com/innovacion.php

Real Academia Española (2014). Diccionario de la lengua española. Madrid, España: Autor.

Rimari Arias, W. La innovación educativa: Un instrumento de desarrollo. Recuperado de http://www.uaa.mx/ direcciones/dgdp/defaa/descargas/innovacion_educativa octubre.pdf
Rogers, E. M. \& Shoemaker, F. F. (1971). Comunication of innovation: A cross-cultural approach. New York, Estados Unidos: The Free Press.

Strutton, D. H.; Lumpkin, J. R. y Vittel, D. J. (1982). Anapplied investigation of Rogers and shoe maker's perceived innovation attributet y pology when marketing to eldery consumers. Journal of Business Research, 10(1), 118-131.

Web and Macros (2015). Definición y concepto de innovación. Recuperado de http://webandmacros.com/ innovacionconceptos.htm 